\title{
Is it a case series? Comment on "Efficacy of modified FOLFOX6 chemotherapy for patients with unresectable pseudomyxoma peritonei"
}

\author{
Min $\mathrm{Luo}^{1} \cdot$ Yetao $\mathrm{Li}^{2}$ (1)
}

Received: 5 October 2020 / Accepted: 10 November 2020 / Published online: 1 January 2021

(c) Japan Society of Clinical Oncology 2021

\section{Dear Editor}

Recently, with great interest, we read the paper "Efficacy of modified FOLFOX6 chemotherapy for patients with unresectable pseudomyxoma peritonei" from Chikashi Ishioka et al. [1]. In this study, the researchers retrospectively evaluated the efficacy and safety of a chemotherapeutic regimen with 5-fuorouracil and oxaliplatin (modified FOLFOX6, mFOLFOX6) for patients with unresectable pseudomyxoma peritonei. It was a valuable experience for the treatment of this rare malignancy. However, the authors declared that "this is the first case series of mFOLFOX6 in patients with unresectable pseudomyxoma peritonei and the first case series of systemic chemotherapy for Asian patients with unresectable pseudomyxoma peritonei". It might be not suitable to classify the study as a case series.

According to the description of Olaf M. Dekkers et al. [2], there are 2 types of case series:

Type 1: A study describing patients with both a specific exposure and a specific outcome. It is important to emphasize the meanings of "both" and "specific". In this study, "modified FOLFOX6 chemotherapy for patients with unresectable pseudomyxoma peritonei" is a specific exposure. However, it is not the case that "a specific outcome" existed in this paper. For example, five patients died, and two survived. Thus this study cannot be classified as a type 1 case series.

Type 2: A study based only on a specific outcome, regardless of whether there are specific exposures. The case group

Yetao Li

taw77@163.com

1 Oncology Department, Guizhou Provincial People's Hospital, Guiyang City 550002, Guizhou Province, China

2 Department of Cardiovascular Surgery, Guizhou Provincial People's Hospital, No.83, East Zhongshan Road, Guiyang City 550002, Guizhou Province, China in a case-control study can be categorized as this type of case series. It is apparent that this research was not based on a specific outcome, as mentioned above. If the paper analyzed the patient characteristics and/or treatment regimens of the five died patients, it would be a type 2 case series.

In summary, sampling is based on a specific outcome in a case series. In this paper, the sampling of the participants was based on a specific exposure, "unresectable pseudomyxoma peritonei" and "modified FOLFOX6 chemotherapy". Participants were followed over time to assess the occurrence of the outcomes. It is the basic characteristic of a cohort study to sample the participants based on exposure, as described by Olaf M. Dekkers et al. [2]. Therefore, this study is a retrospective cohort study rather than a retrospective case series.

In conclusion, this research is not a retrospective case series, but a retrospective cohort study. However, the defects do not obscure the virtues of this paper.

\section{Compliance with ethical standards}

Conflict of interest The authors declare that they have no conflict of interest.

\section{References}

1. Hiraide S, Komine K, Sato Y et al (2020) Efficacy of modified FOLFOX6 chemotherapy for patients with unresectable pseudomyxoma peritonei. Int J Clin Oncol 25(4):774-781

2. Dekkers OM, Egger M, Altman DG et al (2012) Distinguishing case series from cohort studies. Ann Intern Med 156:37-40

Publisher's Note Springer Nature remains neutral with regard to jurisdictional claims in published maps and institutional affiliations. 